\title{
Observation of the Strong Temperature Gradient in Detached Plasma by PIC Simulation with Monte Carlo Collision ${ }^{*}$
}

\author{
Theerasarn PIANPANIT ${ }^{1)}$, Seiji ISHIGURO ${ }^{1,2)}$ and Hiroki HASEGAWA ${ }^{1,2)}$ \\ 1) Department of Fusion Science, SOKENDAI, 322-6 Oroshi-cho, Toki 509-5292, Japan \\ 2) National Institute for Fusion Science, 322-6 Oroshi-cho, Toki 509-5292, Japan
}

(Received 30 November 2015 / Accepted 9 February 2016)

\begin{abstract}
In this work, the $1 \mathrm{D}-3 \mathrm{~V}$ particle simulation with Monte Carlo collision and cumulative scattering angle Coulomb collision was developed to study the kinetic behavior of the detached plasma. The constant pressure and temperature of the neutral gas box in front of the divertor target were used in the simulation. The simulation was performed with a fixed upstream plasma density $n_{\mathrm{u}} \sim 3 \times 10^{18} \mathrm{~m}^{-3}$ and a modified large Coulomb collision frequency. When the neutral gas pressure is increased, there is a strong decrease in the electron temperature inside the neutral gas box. The electron temperature in front of the divertor target also decreases to below $1 \mathrm{eV}$, which is one of the conditions for detached plasma.
\end{abstract}

(C) 2016 The Japan Society of Plasma Science and Nuclear Fusion Research

Keywords: detached plasma, particle simulation, scrape-off layer, parallel transport, divertor

DOI: $10.1585 /$ pfr. 11.2403040

\section{Introduction}

Detached plasma [1] is a method of reducing the heat load to the divertor target during the long operation of future fusion devices. During the detached state, the plasma temperature and particle heat flux decrease significantly. The detached state is achieved when there is a high neutral gas pressure in front of the divertor and spreads the local heat load to a larger area owing to the free moving neutral gas atoms. In experiments, the detached plasma occurs when there is high recycling rate near the divertor plate. This high recycling increases the ion-neutral collision rate and decreases the plasma temperature. Neutral gas puffing can also increase the neutral density near the divertor and cause the plasma temperature to decrease. Impurities are sometimes injected for radiative loss.

Linear devices have been used to study the heat load of plasma to the divertor target by inserting the neutral gas chamber between the plasma source and the target. Many experimental results show a strong decrease in the heat flux to the target when the gas pressure inside the chamber increases $[2,3]$. The linear devices can help in studying the basic physics of the detached plasma and improve the simulation models of the detached plasma.

Most of the detached plasma simulations are performed by fluid models that are based on Maxwellian distribution. Thus, they might be inadequate near the divertor plate [4]. Fluid models also assume a short collision mean free path compared to the system size. However, for some fusion devices, the collision mean free path can be compared to the connection length and thus the kinetic effect

author's e-mail: theerasarn.pianpanit@nifs.ac.jp

*) This article is based on the presentation at the 25th International Toki Conference (ITC25). becomes important.

In this work, particle simulations were performed to investigate the kinetic effect of the plasma. The system has been set up to resemble the linear device. A neutral gas box with constant neutral gas pressure and temperature has been set in front of the divertor target without any influence from the neutral recycling from the wall. The preliminary results suggest that the plasma temperature strongly decreases after increasing the neutral gas pressure and that detached plasma was created. The numerical method that was used in this work is described in Sec. 2. The simulation parameters and results are given in Sec. 3. In Sec. 4, the discussion and future work direction are presented.

\section{Numerical Method}

This simulation consists of the particle-in-cell (PIC) and collision parts. The PIC simulation solves the equation of motion for individual particle inside the plasma and finds the self-consistent electric field. The collision part includes the Monte Carlo collision (MCC) with neutral particles and the Coulomb collision. MCC is necessary for the atomic processes such as charge exchange, ionization, and excitation. Physical processes from gas puffing can also be included in the MCC. In low temperature and high density plasma, Coulomb collisions become important and the PIC simulation alone cannot accurately simulate the Coulomb collisions, which contribute to the detached plasma. The system configuration is divided into the plasma source region, the source free region, and the neutral source region (Fig. 1). Inside the system, the magnetic field is uniform and pointing toward the collector plate. 


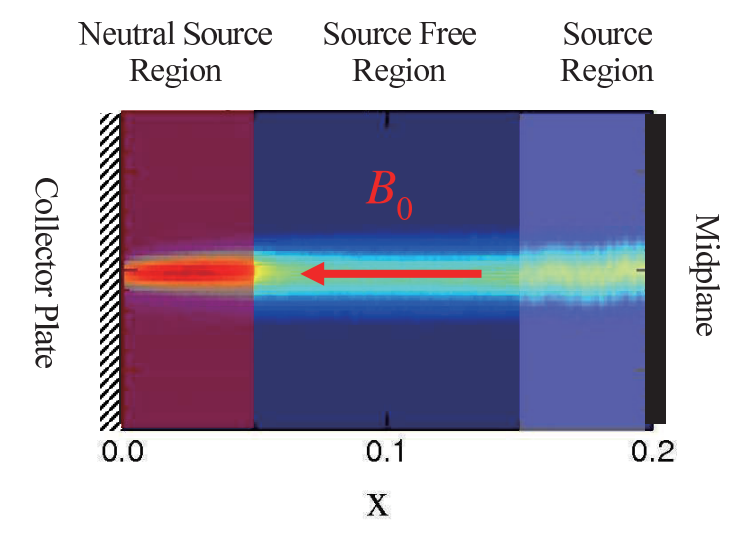

Fig. 1 System configuration with the plasma source on the righthand side and the collector target on the left-hand side; plasma flow is from the right- to the left-hand side.

\subsection{Boundary conditions}

The boundary conditions of the system are defined for the left- and right-hand side of the system box. If $x$ is the position along the field line and $L$ is the system length, $x=$ $L$ at the right-hand side of the system is set as the symmetry midplane, and the particles are reflected back to the system. The left-hand side is the collector plate $(x=0)$, which is assumed to fully absorb the particles and accumulate the charge density $\sigma$.

The electrostatic potential at the midplane is set to zero, or

$$
\phi(x=L)=0 .
$$

The electric field at the collector plate depends on the charge density at the plate and can be written using Gauss's law as

$$
E(x=0)=\sigma / \varepsilon_{0},
$$

where $\varepsilon_{0}$ is the permittivity of free space. This equation can be also written in the finite difference form, as in Ref. 5.

\subsection{Particle source}

The uniform particle source is placed in the right-hand side of the system. Let us define $v_{\|}$and $v_{\perp}$ as the parallel and perpendicular particle velocity, respectively. $m$ is the particle mass and $T_{\mathrm{s}}$ is the source temperature. The distribution function of the velocity in the parallel direction to the magnetic field line for the particle source is $f\left(v_{\|}\right) \sim$ $v_{\|} \exp \left(m v_{\|}^{2} / 2 T_{\mathrm{s}}\right)[6,7]$. In the perpendicular direction, the distribution function becomes $f\left(v_{\perp}\right) \sim \exp \left(m v_{\perp}^{2} / 2 T_{\mathrm{s}}\right)$ or the Maxwellian distribution function.

\subsection{Monte Carlo collision with neutral par- ticle}

In the MCC simulation, the null collision method has been used [10]. In null collision method, the maximum collision probability is calculated instead of calculating every particle collision probability. Therefore the maximum

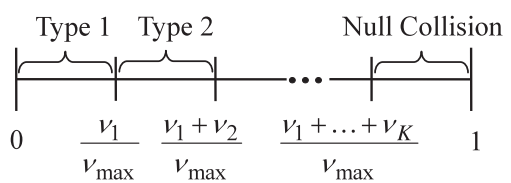

Fig. 2 Collision probability for $K$ types of atomic processes in the null collision method.

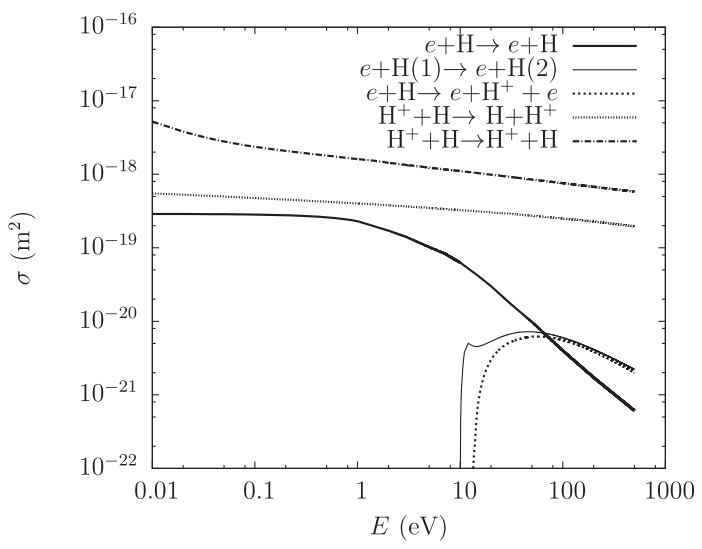

Fig. 3 Cross sections of different types of atomic processes [8, 9].

probability of the particle collision is

$$
P_{\text {null }}=1-\exp \left[-\max _{\mathbf{x}, v}\left(n_{\mathrm{n}} \sigma_{\mathrm{T}} v\right) \Delta t\right]
$$

where $n_{\mathrm{n}}$ is the density of the neutral particle, $\sigma_{\mathrm{T}}$ is the total cross section of the atomic processes of each species, $v$ is the speed of each particle, and $\Delta t$ is the time interval of each time step. This probability defines the number of particles that collide in each time step and the collision type is selected for each particle, as in Fig. 2, where $v=n_{\mathrm{n}} \sigma v$ is the collision frequency with the probability that the selected particle will not collide with the neutral particle. This is the null collision region in Fig. 2.

The collision processes that are included in the simulation are divided into two groups, which are the electrons and hydrogen ions group in our case. In the electrons group, the atomic processes are elastic collision, ionization, and excitation. By using the null collision method, the number of collision types is $K=3$. In the hydrogen ions group, the atomic processes are elastic collision and charge exchange. Thus, the number of collision types is $K=2$. The cross section data are shown in Fig. 3 .

\subsection{Coulomb collision}

In this simulation, the cumulative scattering angle binary collision was applied to the Coulomb collision process [11]. Rather than calculate the small scattering angles of the binary collision of each particle in small time steps [12], we group the scattering angles into a large cumulative scattering angle.

In Nanbu's Coulomb collision model [11], the small 
scattering angles at each time step can be treated as the cumulative scattering angle. If a test particle starts with relative velocity to a field particle $\mathbf{g}_{0}$, after the $1 \mathrm{st}, 2 \mathrm{nd}$, $\ldots$, Nth collisions, the relative velocities are $\mathbf{g}_{1}, \mathbf{g}_{2}, \ldots, \mathbf{g}_{N}$. The cumulative scattering angle $\chi_{N}$ is defined as the angle between $\mathbf{g}_{0}$ and $\mathbf{g}_{N}$ or

$$
\cos \chi_{N}=\mathbf{g}_{0} \cdot \mathbf{g}_{N} / g^{2} \text {. }
$$

Because the Coulomb collision is elastic, the speed relative to the fixed field particle must be conserved, thus $g=g_{1}=$ $g_{2}=\ldots=g_{N}$.

To find the cumulative scattering angle for a test particle, Nanbu introduced the isotropy parameter $s$

$$
s=n_{\mathrm{t}} g \pi b_{0}^{2}(\ln \Lambda) \Delta t,
$$

where $n_{\mathrm{t}}$ is the target particle density, $g$ is the relative speed of two colliding particles, and $b_{0}$ is the classical distance of the closest approach. $s$ is used to determine constant $A$ in the following equation:

$$
\operatorname{coth} A-A^{-1}=e^{-s} \text {. }
$$

The above equation needs to be calculated in advance and saved to an array to reduce the computational time. By using this constant $A, \chi_{N}$ is calculated from the following equation:

$$
\cos \chi_{N}=\frac{1}{A} \ln \left(e^{-A}+2 R \sinh A\right),
$$

where $R$ is a random number from 0 to 1 .

\section{Simulation Results}

The simulation is carried out with the system parameters in Table 1. The neutral gas box is in the left-hand side of the system and the plasma source region is in the righthand side of the system as in Fig. 1. The lengths of the neutral source and plasma source regions are listed in Table 1. In this work, the neutral gas dynamics was not included. The neutral gas temperature and density are assumed constant throughout the simulation and both are constant along the $x$-axis inside the neutral source region. We also assume that the neutral gas density becomes zero outside the neutral source region.

At the initial time step, the injection rate of the ion and electron pair is constant but the simulation is carried out with fixed upstream density. Thus, when $n_{\mathrm{u}}>3 \times 10^{18} \mathrm{~m}^{-3}$, no particles are injected to the system and the particles inside the source region are heated. The number of particles to be heated is equal to the number of particles that are injected. The velocity of the particles that are heated inside the source region is randomly changed to the particle source velocity without changing the direction. This process heat the particles at the upstream region in order to fix the upstream temperature.

Owing to the low plasma density in the simulation, the detached state cannot be observed without modifying the
Table 1 Simulation parameters.

\begin{tabular}{ll}
\hline System length & $L=0.2 \mathrm{~m}$ \\
Mass ratio & $m_{\mathrm{i}} / m_{\mathrm{e}}=1836$ \\
Electron source temperature & $T_{\mathrm{e} 0}=10 \mathrm{eV}$ \\
Ion source temperature & $T_{\mathrm{i} 0}=10 \mathrm{eV}$ \\
Particle injection rate & $R_{\mathrm{inj}}=0.5$ super particle \\
$\quad$ Initial time) & \multicolumn{1}{c}{ pairs per $\Delta t$} \\
Super particle size & $N_{\mathrm{S}}=1 \times 10^{11}$ \\
Plasma source length & $L_{\mathrm{S}}=0.05 \mathrm{~m}$ \\
Neutral source length & $L_{\mathrm{n}}=0.05 \mathrm{~m}$ \\
Neutral gas temperature & $T_{\mathrm{n} 0}=0.026 \mathrm{eV}$ \\
Magnetic field & $B_{0}=1 \mathrm{~T}$ \\
\hline
\end{tabular}

(a)

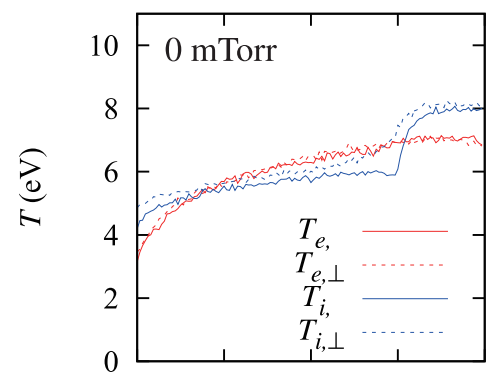

(b)

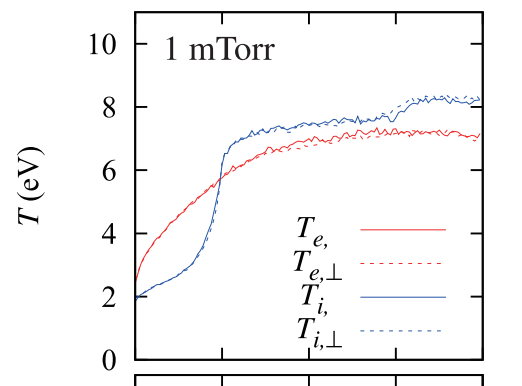

(c)

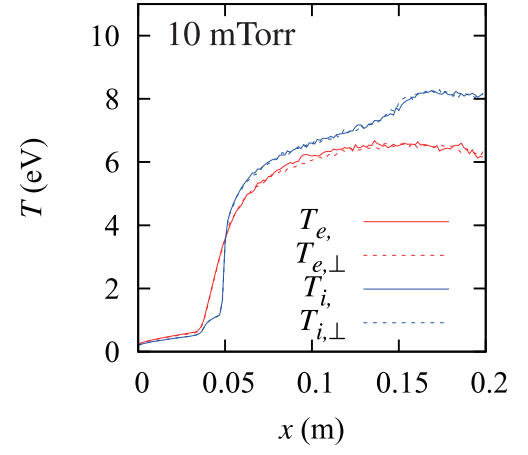

Fig. 4 Parallel and perpendicular temperature profiles of electrons and ions at $t=24 \mu \mathrm{s}$ for different neutral gas pressures: (a) $P_{N}=0$ mTorr (b) $P_{N}=1$ mTorr, and (c) $P_{N}=$ $10 \mathrm{mTorr}$.

Coulomb collision frequency. The density threshold for plasma detachment has been observed in the linear plasma device [13]. In high density plasma, the detachment can be easily observed and the observation suggests that the electron and ion collision needs to be sufficiently large for the electrons to transfer energy to ions. In this simulation, the system size and plasma density are much less than the condition for the detached plasma; thus, the Coulomb collision frequency is modified to $v_{\mathrm{c}}=100 v_{\mathrm{c} 0}$. The collision 
(a)

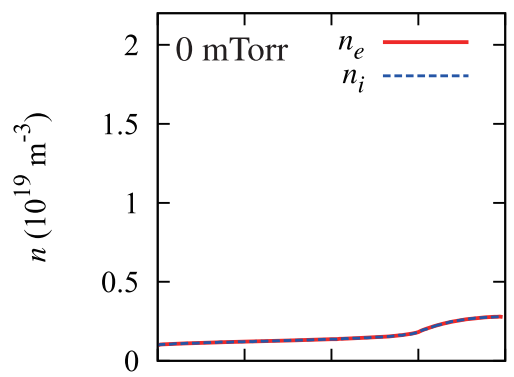

(b)

(c)

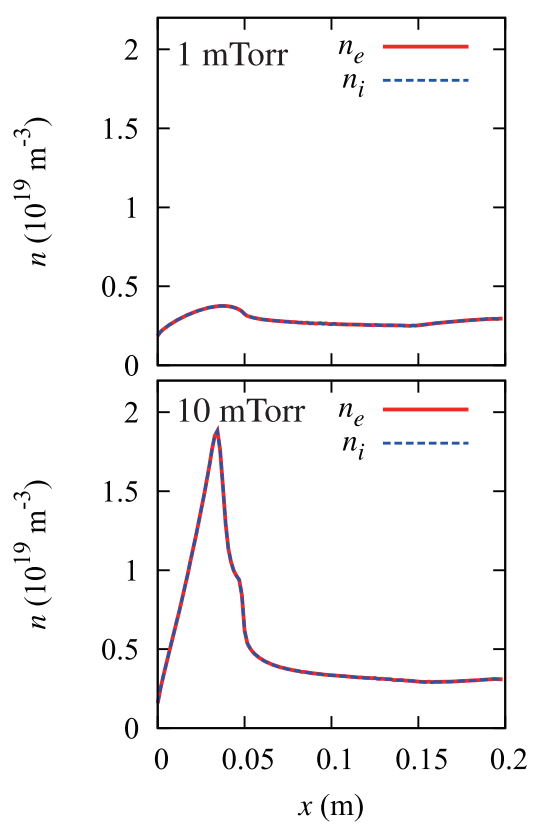

Fig. 5 Density profile of electrons and ions at $t=24 \mu$ s for different neutral gas pressures: (a) $P_{N}=0 \mathrm{mTorr}$ (b) $P_{N}=$ $1 \mathrm{mTorr}$, and (c) $P_{N}=10 \mathrm{mTorr}$.

frequency can be modified by Eq. 3, where the isotropy parameter depends on the collision frequency $s \sim v_{\mathrm{c} 0} \Delta t$ and is modified to $s^{\prime} \sim v_{\mathrm{c}} \Delta t$.

The results are shown in Figs. 4 and 5. The figures show the decrease in plasma temperature and the increase in plasma density inside the neutral gas box. Energy is transferred from the electron to ion and most of the energy loss is caused by the ion elastic collision and charge exchange.

The total plasma pressure profiles are shown in Fig. 6. From the momentum conservation of the 1D fluid equation [14], if there is no collision between the plasma and neutral gas, the total plasma pressure $P_{\text {tot }}$ along the flux tube must be constant or the following equation:

$$
P_{\mathrm{tot}}=P+m_{\mathrm{i}} n V^{2}=\text { constant, }
$$

where $P=n_{\mathrm{i}} k T_{\mathrm{i}}+n_{\mathrm{e}} k T_{\mathrm{e}}$ and $V$ is the average velocity of the plasma. At $P_{N}=0 \mathrm{mTorr}$, the total plasma pressure is constant along the $x$-direction. When the neutral gas pressure increases, the total plasma pressure drops in front of the divertor target. The total plasma pressure at $x=0$ approaches zero at $P_{N}=10$ mTorr.

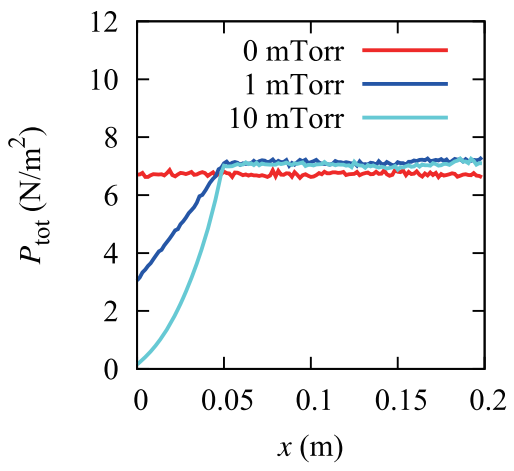

Fig. 6 The total plasma pressure profile at $t=24 \mu$ s for different neutral gas pressure.

\section{Summary and Discussion}

The results suggest a strong temperature gradient inside the neutral gas region. The plasma temperature decreases below $1 \mathrm{eV}$ and the temperature approaches the neutral gas temperature when the neutral gas pressure increases to approximately 10 mTorr. The total pressure also decreases sharply inside the neutral gas box. The detached plasma can be observed by using the PIC-MCC simulation with the modified Coulomb collision frequency.

In this simulation, the plasma density increases strongly inside the high pressure neutral gas region, and recombination can become important in decreasing the particle flux to the divertor target during the detached state. There are many theoretical models and experimental results that suggest that recombination plays an important role in describing the strong decrease in the particle flux of the detached plasma $[15,16]$. Thus in the future, we plan to implement the recombination process in the simulation [17]. Furthermore, this type of simulation can be used to understand the sheath formation in front of plasma facing materials and can be used to calculate the ion and electron heat flux directly.

\section{Acknowledgements}

This work was performed with the support and under the auspices of the NIFS Collaboration Research programs NIFS14KNXN279 and NIFS14KNSS059. The simulation was carried out by the Plasma Simulator at NIFS and the HELIOS supercomputer system at the Computational Simulation Centre of the International Fusion Energy Research Centre (IFERC-CSC), under the Broader Approach collaboration between Euratom and Japan, implemented by the Fusion for Energy and JAEA.

[1] G.F. Matthews, J. Nucl. Mater. 220-222, 104 (1995).

[2] W.L. Hsu, M. Yamada and P.J. Barrett, Phys. Rev. Lett. 49, 1001 (1982).

[3] N. Ohno et al., Nucl. Fusion 41, 1055 (2001).

[4] O.V. Batishchev et al., Phys. Plasmas 4, 1672 (1997).

[5] W.S. Lawson, J. Comput. Phys. 80, 253 (1989).

[6] G.A. Emmert et al., Phys. Fluids 23, 803 (1980). 
[7] R.J. Procassini, C.K. Birdsall and B.I. Cohen, Nucl. Fusion 30, 2329 (1990).

[8] R.K. Janev, Atomic and Molecular Processes in Fusion Edge Plasmas (Plenum Press, New York, 1995).

[9] R.K. Janev, D. Reiter and S. Samm, Collision Processes in Low-Temperature Hydrogen Plasmas (JUEL-4105, 2003).

[10] V. Vahedi and M. Surendra, Comput. Phys. Commun. 87, 179 (1995).

[11] K. Nanbu, Phys. Rev. E 55, 4642 (1997).
[12] T. Takizuka and H. Abe, J. Comput. Phys. 25, 205 (1977).

[13] N. Ezumi et al., J. Nucl. Mater. 241-243, 349 (1997).

[14] P.C. Stangeby, The Plasma Boundary of Magnetic Fusion Devices (Taylor \& Francis Group, New York, 2000) p.399.

[15] S.I. Krasheninnikov et al., Phys. Plasmas 4, 1638 (1997).

[16] N. Ohno et al., Phys. Rev. Lett. 81, 818 (1998).

[17] K. Nanbu and K. Denpoh, J. Phys. Soc. Jpn. 67, 1288 (1998). 